\title{
An algorithm of lips secondary positioning and feature extraction based on $\mathrm{YCbCr}$ color space
}

\author{
SHEN Xian-geng ${ }^{1}$, WU Wei $^{2}$ \\ ${ }^{1}$ Engineering University of CAPF, Postgraduate Brigade, xi'an 710086, China; \\ ${ }^{2}$ Engineering University of CAPF, Dept. of Information Engineering, xi'an 710086, China
}

Keywords: face recognition; lips; YCbCr; MATLAB; feature extraction

\begin{abstract}
In recent years, face recognition is becoming one of the hot spots in the field of human-computer interaction. As the main organ, lips are one of the important features of face recognition. In order to get the features of lips, the paper positions the lips and segments it. The paper simulates the process by the MATLAB and gains the features of lips. Compared to the traditional algorithm of RGB color space, it has the advantages of fast calculation and high accuracy rate. It also gains the good effect in the light of different level.
\end{abstract}

\section{Introduction}

With the era development, identity recognition technology is becoming a more and more important research topic. Face recognition is an important research in the area of identification, which plays an important role in the intelligent buildings, intelligent monitoring and other fields. Lips, as one of the facial features, and in the central position of the face, is an important part of the face recognition. At present there are two main types of lip feature extraction methods at home and abroad. One method is based on pixels, namely the lips first image is converted to a grayscale image, using the gray information as characteristic vector of the lips[1]. Such as the direct pixel which is proposed by Yuhas ,the vector quantization method, the lip method of the university of karlsruhe in Germany and the MIT media lab Mase and Pentland adopts the method based on optical flow.The second method is based on the model, the method of the lip contour structure to establish a model, realizing the characteristics of the lips which based on the template matching method.

At present, the major problems affecting lips recognition are as follows[2]: (1)Different person's lips shapes vary, and are easy affected by light and head movement, the influence of such factors as great influence on the lip contour extraction. (2)Because the lip color and skin color differences in the RGB color space is small, only in G there is a significant difference on the weight, it influents the lip feature extraction effect. (3) The template is complex, the construction of a computational cost is larger.(4) Lips with poor robustness. In different facial expressions, the shape of the lips, causing the lips with poor robustness, affecting the effects of the feature extraction of labial ministry.

Algorithm is in YCbCr color space, this paper uses dynamic threshold to the secondary positioning of the lips, for the localization of lip shape of image segmentation and edge detection, then gets the characteristics of the lips.

\section{Feature extraction method and steps}

Lip color is one of the most important features of the lips, in RGB color space, color and brightness information are mixed together, so in the lip color detection is easily affected by light detection. YCbCr color space will separate the brightness from the face image information. The shade of the image information totally included in the $\mathrm{Cb}$ and $\mathrm{Cr}$ components. So you can avoid the recognition error caused by uneven brightness[3]. In this paper, according to the good clustering effect in YCbCr color space, removing the lip extraction error caused by uneven illumination, getting the feature of the lips by using the secondary positioning of color face image feature extraction and YCbCr color space. The steps are as follows: 
step1: First of all, we do the segmentation for the face image which is already positioned[4]. As shown in figure 1,2 and3.

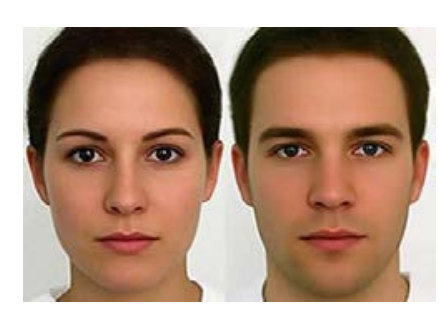

Fig.1: The face image before the positioning

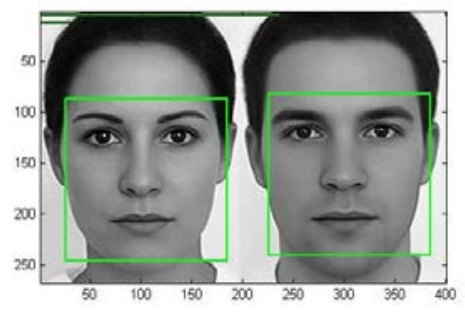

Fig.2: The face image after the positioning

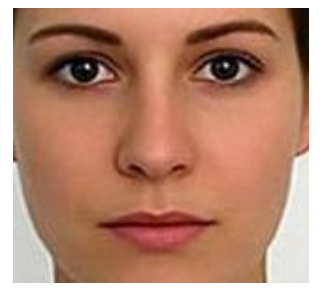

Fig.3: Face image of segmentation

Step2: Due to lips located roughly under a third, three 5 middle place of the face, so we deal with the face image and get a preliminary image of lips[5]. As shown in figure 4.

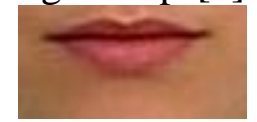

Fig.4: Preliminary image of lips

Step3: Using YCbCr color space and dynamic threshold algorithm realizes the lip area secondary division.

Step4: Optimized lip feature area and extract the feature points through edge detection and corrosion expansion.

\section{Secondary segmentation and feature extraction of the lips}

According to the diversity of lip color and skin color of $\mathrm{Cb}$ and $\mathrm{Cr}$ in $\mathrm{YCbCr}$ color space, widen the difference of $\mathrm{Cb}$ and $\mathrm{Cr}$ in $\mathrm{YCbCr}$ color space, through dynamic threshold value to judge the lip pixels to get the second division coordinates of lips.

Lip color and skin color in RGB and clustering effect differences in YCbCr. RGB color space is a kind of commonly used color space, with red, green, and blue components. YCbCr color space is a kind of color space, it usually used in the film or digital camera system[6]. Through to the lip color and skin color in RGB and YcbCr, we can find that lip color and skin color clustering effect in YcbCr color space is better than clustering effect in RGB color space. As shown in figure 5and6.

According to the histogram we can know that ,the lip color and skin color pixels are centered in the YCbCr color space, the clustering effect is better than in RGB space. Because the lips of $\mathrm{Cb}$ and $\mathrm{Cr}$ component are slightly larger than the skin of $\mathrm{Cb}$ and $\mathrm{Cr}$ components, by $\mathrm{Cb}, \mathrm{Cr}$ components together, increase the difference between lips and skin, and then identify the lips. 

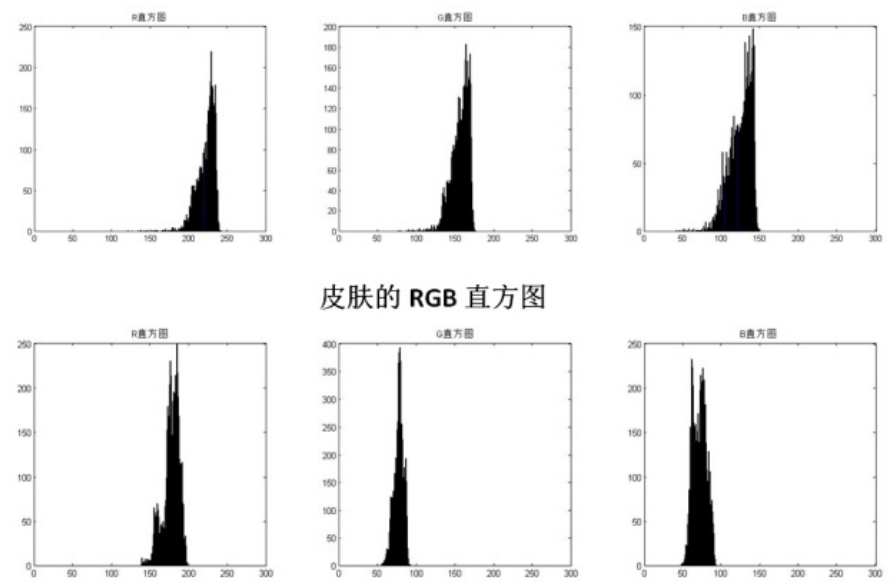

皮肤的 RGB 直方图
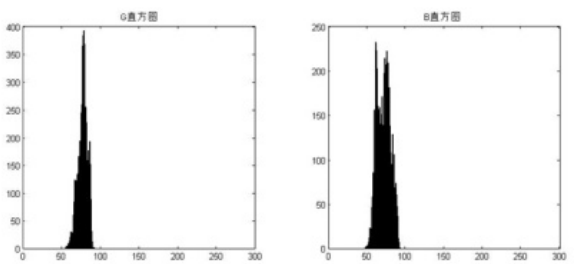

嘴唇的 RGB 直方图

Fig.5: Lip color and color RGB histogram
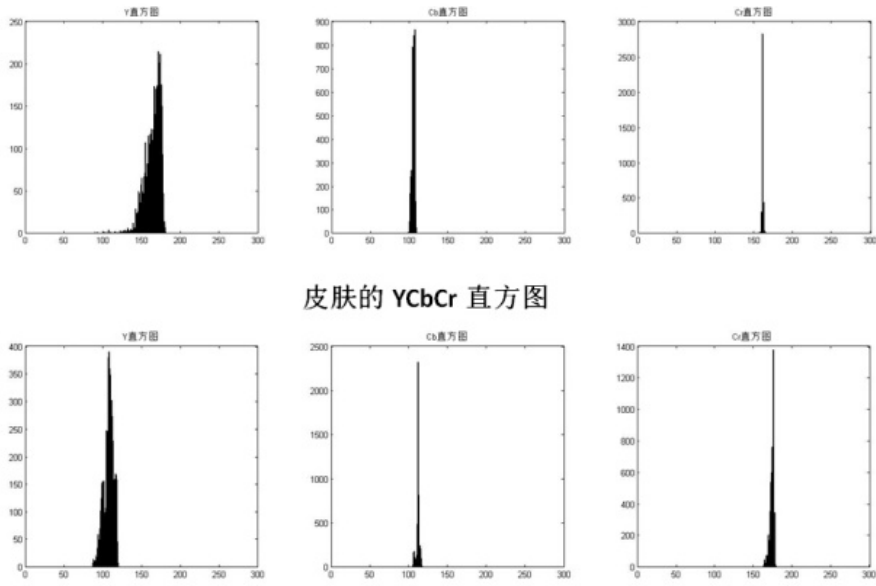

皮肤的 $\mathrm{YCbCr}$ 直方图
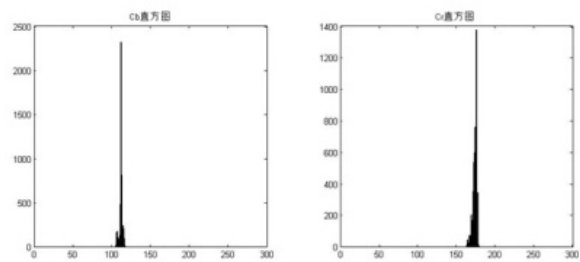

嘴唇的 $\mathrm{YCbCr}$ 直方图.

Fig.6: Lip color and color YCbCr histogram

The determination of the threshold. In order to eliminate the influence of light intensity on the lip color, in this paper, the algorithm adopted the luminance component in YCbCr space ,using $\mathrm{Cb}$ and $\mathrm{Cr}$ component in operations. Firstly calculates the average of $\mathrm{Cb}$ component $\mathrm{t}_{1}$, According to figure 6, lip color and color of skin of Cb component are more than 100, Cr's weight are more than 150. In order to prevent the interference of other pixels, so take a lip color $\mathrm{Cb}$ components more than 100 points and $\mathrm{Cr}$ above 150 points. Set $S_{1}$ is the sum of Cb weight greater than 100 for your lips and variable. $i$ for the pixel values of pictures. $n_{i}$ for the number of pixels who's value is $i$. $N$ for the number of pixels who's grey value is greater than a certain gray level. The Cb weight average can be calculated by the following formula:

$$
\begin{gathered}
N=\sum_{i=100}^{255} n_{i} \\
S_{1}=\sum_{i=100}^{255} n_{i} *_{i} \\
t_{1}=\frac{S_{1}}{N}
\end{gathered}
$$

In the same way, set s for $\mathrm{Cr}$ weight is greater than 150 . The $\mathrm{Cr}$ average weight can be calculated by the following formula:

$$
N=\sum_{i=150}^{255} n_{i}
$$




$$
\begin{aligned}
& S_{2}=\sum_{i=150}^{255} n_{i} * i \\
& t_{2}=\frac{S_{2}}{N}
\end{aligned}
$$

In the end, we can get the resulting threshold thresh $1=\frac{t_{1}+t_{2}}{2}$ and the threshold thresh2 $=t_{2}$.Then calculate the lips in the initial segmented regions at the same time meet the points $\frac{C b+C r}{2}>$ thresh 1 and $C r>$ thresh 2 ,then do binarization to pixel. Get the binary image of lip area. As shown in figure 7.

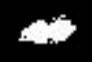

Fig.7: Binary image of lip area

The second division of lips. Doing the secondary positioning to the lip image by using the lip shape left and right sides is symmetrical, the characteristics of the narrow middle wide on both sides. First of all, we should determine lips secondary segmentation region boundary. According to the binary image of the lips for horizontal and vertical projection, draw the lip area of horizontal and vertical histogram. As shown in figure 8 and 9.

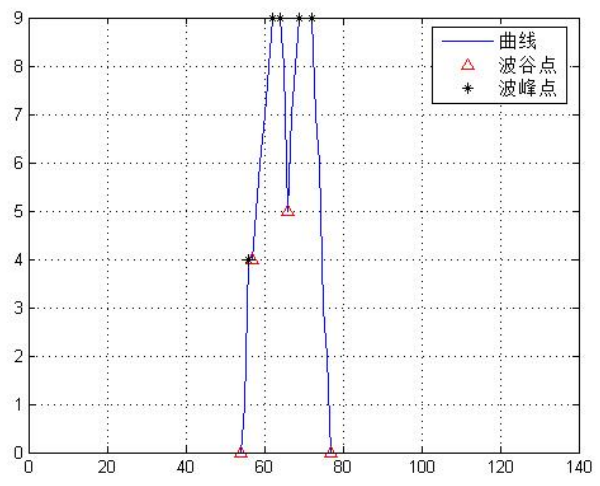

Fig.8: The horizontal projection of lips

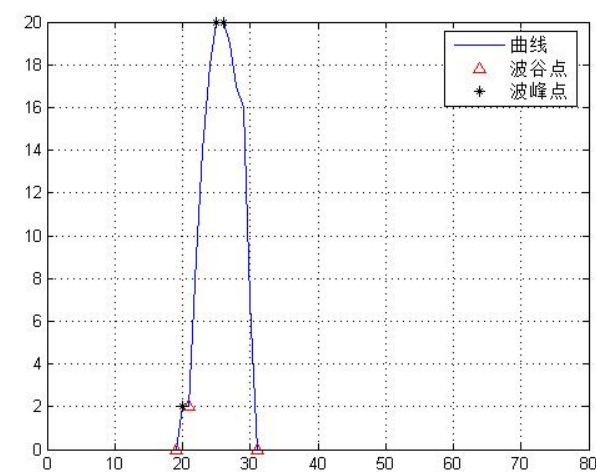

Fig.9: The vertical projection of lips

Then find out the lips horizontal projection zero $X_{1}, X_{2}$ They are the critical point of lips' two sides. The point between the two peaks of low is abscissa of the central place of your lips. Calculate the horizontal distance between two zero point and the central point $L_{1}=X-\mathrm{X}_{1}\left(L_{2}=X_{2}-X\right)$. Get big zero distance and increase the distance of 10. $L=L_{1}+10\left(L=L_{2}+10\right), L$ as a half long of lips horizontal interception. The central trough point is the starting point of the cross on the left for 
transverse segmentation. Similarly find zero vertical projection $Y_{1}, Y_{2}$ and the vertices $Y$. Calculate the vertical distance from $Y_{1}, Y_{2}$ to $Y$. The distance is $H_{1}=Y-Y_{1}\left(H_{2}=Y_{2}-Y\right)$. Take big zero distance and increase the distance of $8 H=H_{1}+8\left(H=H_{2}+8\right) . H$ as the semimajor vertical intercept of lips. The vertices of the ordinate is the starting point of the vertical segmentation for your lips. And then get the secondary segmentation image segmentation of lips. As shown in figure 10.

Fig.10: The second image segmentation of lips

The feature extraction of lips. Disposing the lips secondary image segmentation by using RGB color space red discriminant method. And get the edge of image by Laplace. And then to corrosion, expansion and filling morphology processing, etc. Remove the hole of the edge and noise in the image. We can better detect the shape of the lips. As shown in figure 11 and 12.

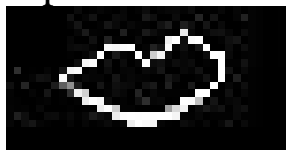

Fig.11: The image of lips edge detection

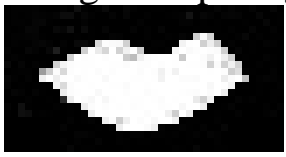

Fig.12: The image after dealing with the morphology

Through the experiment results show that secondary positioning feature extraction algorithm can extract the edge of the lip image clearly, and can detect the lip contour. According to feature of lips, we can get the feature point of lower lip and the upper lip. As shown in figure 13 and 14.

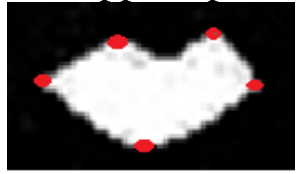

Fig.13: The feature point position of binary image

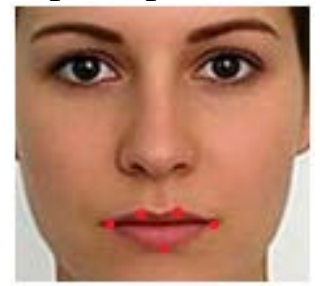

Fig.14: The feature point position of lips

Through the integration of the gray-scale projection and edge detection and so on, dealing with the face image to get three feature points of the nose[7][8]. As shown in figure 15.

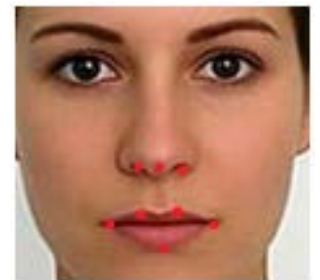

Fig.15: The feature point position of nose

Set the coordinates of the corners of the mouth around $\left(x_{1}, y_{1}\right)$ and $\left(x_{2}, y_{2}\right)$, Lower lip coordinates for $\left(x_{3}, y_{3}\right)$, the coordinates of upper lip around $\left(x_{4}, y_{4}\right)$ and $\left(x_{5}, y_{5}\right)$, The coordinates of the feature points of nose around $\left(x_{6}, y_{6}\right),\left(x_{7}, y_{7}\right)$ and $\left(x_{8}, y_{8}\right)$.Then calculating the feature vector of lower lip: $A_{1}=\left(x_{1}-x_{3}, y_{1}-y_{3}\right), A_{2}=\left(x_{2}-x_{3}, y_{2}-y_{3}\right)$. The feature vector of upper lip: $A_{3}=\left(\mathrm{x}_{4}-\mathrm{x}_{1}, \mathrm{y}_{4}-\mathrm{y}_{1}\right), A_{5}=\left(\mathrm{x}_{5}-\mathrm{x}_{2}, \mathrm{y}_{5}-\mathrm{y}_{2}\right)$. The feature vector of corners of mouth: 
$A_{6}=\left(\mathrm{x}_{6}-\mathrm{x}_{1}, \mathrm{y}_{6}-\mathrm{y}_{1}\right), A_{7}=\left(\mathrm{x}_{7}-\mathrm{x}_{1}, \mathrm{y}_{7}-\mathrm{y}_{1}\right), A_{8}=\left(\mathrm{x}_{7}-\mathrm{x}_{2}, \mathrm{y}_{7}-\mathrm{y}_{2}\right), A_{9}=\left(\mathrm{x}_{8}-\mathrm{x}_{2}, \mathrm{y}_{8}-\mathrm{y}_{2}\right)$. When it used to identify the lips, calculating the vector Angle between the feature of recognition and the feature in face database. If the Angle within the scope of the permit, then identify the lips for a certain object in face library, and then complete identity recognition[9].

\section{The algorithm simulation}

According to the algorithm proposed in this paper. By using MATLAB powerful matrix operations, we do the feature extraction to the face images under the different light intensity and Angle. And with the current red method which are used to get the lip contour feature points[10]. As shown in figure 16 and 17.

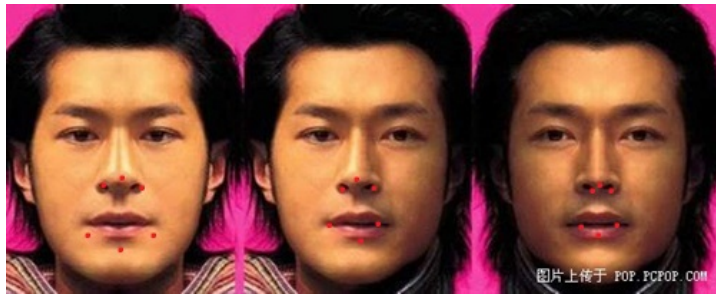

(a) Common method

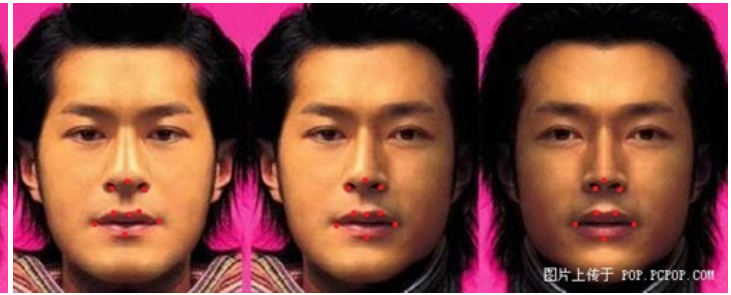

(b) The algorithm of this paper

Fig.16: The lip feature point extraction under different light conditions

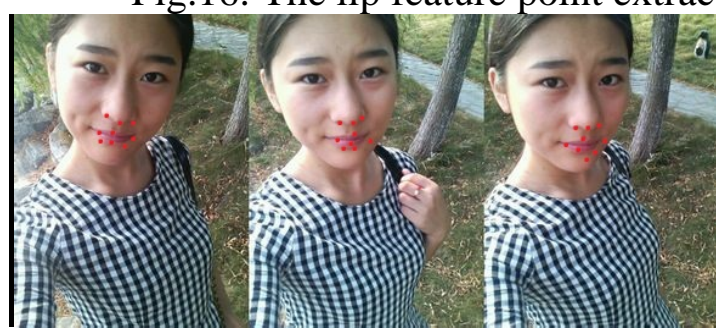

(a) Common method

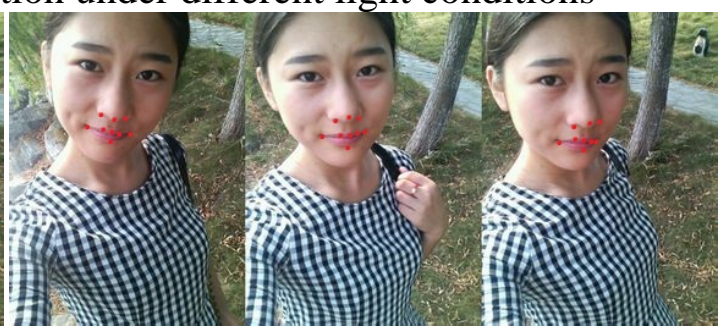

(b) The algorithm of this paper

Fig.17: The lip feature point extraction under different angles

Can be seen by comparing, under different light conditions in the front of the face image can be very good to extract the feature points, the lips in different Angle in the image of a face also can obtain a certain result, lip feature vector calculation laid a foundation for the next step.

\section{Conclusions}

In this paper, based on $\mathrm{YCbCr}$ color space, face the face image, using the secondary positioning algorithm, greatly reducing the influence on the lip feature extraction. Through simulation experiments, under the environment of MATLAB 7 for color face image can better extracted lip feature points. The algorithm computational complexity is small, simple, and except for the influence of uneven illumination of lip feature recognition.

\section{References}

[1] WEI Dong-dong, Technology of Hunan Face Feature Extraction and Recognition. Changsha: Science and Engineering National University of Defense Technology.2006.

[2] DUAN Qi, Comparison of Feature Extraction and Classification Methods for Face Recognition. Shanghai: East China university of science.2012.

[3] YUAN Yan-yan, HUI Xiao-wei, Face Detection Baced on YCbcr Skin Color Model and SNOW Classifier. Computer systems and applications,2010,19(1): 157-160.

[4] Mikael NILLSON. FACE DETECTION USING LOCAL SMQT FEATURES AND SPLIT UP SNOWCLASSIFIER. Sweden: Blekinge Institute of Technology School of Engineering. 2007: 589- 592. 
[5] WANG Xiao-ping, HE Yu-feng. An Automatic Approach to Lip Localization, Contour Extraction and Tracking. PR\&AI, 2007, 4(20):485-491.

[6] ZHANG Ling. Lip Color and Color Difference of Labial Ministry Detection Algorithm Research. computer systems \& applications. 2012,48(30): 183-186.

[7] WU Zheng. Research on Facial Feature Points Extraction and Relevant Application. Shanghai: Shanghai Jiao Tong University.2007.

[8] MA Yan-ni, GENG Guo-hua. Localization and extraction for feature points of human faces. Computer Engineering and Applications. 2009,45(18): 167-170.

[9] LIU Cheng-ming, ZHANG Li-ming. An improved method of facial feature points positioning.Shanghai: Fudan University. 2006,45(4): 452-463.

[10] DU Chun-hua, Research of facial landmark location and face recognition. Shanghai: Shanghai Jiao Tong University.2008. 